\title{
Trombofilias, esterilidad, infertilidad y tratamiento de reproducción asistida
}

\author{
Thrombophilias, sterility, infertility and assisted reproductive treatment \\ Trombofilias, esterilidade, infertilidade e tratamento reprodutivo assistido
}

Virginia Chaquiriand

ORCID: 0000-0003-1509-2780 Ginecóloga. Ex Profesora Adjunta Clínica Ginecotocológica B. Facultad de Medicina. UdelaR.

Resumen: La esterilidad se define como la no obtención del embarazo luego de un año de relaciones sexuales sin protección en parejas heterosexuales. Puede afectar hasta un 15 a 20\% de las parejas en edad reproductiva en algún momento de la vida. Las causas de esterilidad se dividen en: factor masculino, factor femenino, causa mixta y esterilidad sin causa aparente. Se ha planteado que las trombofilias hereditarias y adquiridas, podrían estar involucradas en su etiología; y que las distintas estrategias de tratamiento de éstas (ácido acetil salicílico, heparina de bajo peso molecular, corticoides, hidroxicloroquina, entre otros) podrían mejorar los resultados de las técnicas de reproducción asistida.

Palabras clave: esterilidad, infertilidad, trombofilia.

Abstract: Sterility is defined as failure to achieve pregnancy after one year of unprotected sexual intercourse in heterosexual couples. It can affect up to $15-20 \%$ of couples of reproductive age at some point in life. The causes of sterility are divided into: male factor, female factor, mixed cause and sterility with no apparent cause. It has been suggested that hereditary and acquired thrombophilias could be involved in its etiology; and that the different treatment strategies for these (acetylsalicylic acid, low molecular weight heparin, corticosteroids, hydroxychloroquine, among others) could improve the results of assisted reproductive techniques.

Key words: sterility, infertility, thrombophilia.

Resumo: Esterilidade é definida como o fracasso em engravidar após um ano de relação sexual desprotegida em casais heterossexuais. Pode afetar até $15-20 \%$ dos casais em idade reprodutiva em algum momento da vida. As causas da esterilidade são divididas em: fator masculino, fator feminino, causa mista e esterilidade sem causa aparente. Foi sugerido que trombofilias hereditárias e adquiridas podem estar envolvidas em sua etiologia; e que as diferentes estratégias de tratamento para estes (ácido acetilsalicílico, heparina de baixo peso molecular, corticosteroides, hidroxicloroquina, entre outros) poderiam melhorar os resultados das técnicas de reprodução assistida.

Palavras-chave: esterilidade, infertilidade, trombofilia.

Recibido: 06/02/2021 - Aceptado: 31/05/2021 
La esterilidad se define como la no obtención del embarazo luego de un año de relaciones sexuales sin protección en parejas heterosexuales. Puede afectar hasta un 15 a $20 \%$ de las parejas en edad reproductiva en algún momento de la vida.

Debido a los cambios sociales de nuestra población, es un motivo de consulta cada vez más frecuente, y particularmente en nuestro país, la ley de reproducción 19.167/2013 ha permitido que las parejas o pacientes que necesitan asistencia puedan tenerla dentro de la cobertura del sistema de salud.

Lamentablemente hasta el momento, los resultados en reproducción asistida distan mucho del $100 \%$ de éxito que nos gustaría, lo que hace que muchas veces no tenemos los resultados deseados y continuamente se están investigando causas y buscando alternativas para mejorar los mismos.

Las causas de esterilidad se dividen en: factor masculino, factor femenino, causa mixta y esterilidad sin causa aparente.

El fallo recurrente de implantación, llamado en inglés recurrent implantation failures (RIF) tiene múltiples denominaciones según los autores pero se refiere a los casos de pacientes que realizan múltiples intentos de transferencias de embriones de muy buena calidad, y se transfieren a pacientes que el útero no tiene patología diagnosticada y no se consigue el embarazo. Al día de hoy hay muchos estudios que han mostrado que, en muchos casos, el fallo de implantación puede deberse a alteraciones a nivel cromosómico de los embriones, o a alteraciones en la sincronización entre el embrión y la ventana de implantación, por lo cual se plantea la realización de PGT-A, o de test de evaluación endometrial para mejorar los resultados. A pesar de todo ello, sigue habiendo un grupo de pacientes que no consiguen la implantación, y las causas se desconocen.

Algunos trabajos han postulado que las trombofilias hereditarias $(\mathrm{TH})$ y adquiridas (síndrome antifosfolipídico, SAF), podrían estar involucradas en la etiología de la esterilidad, pérdida de embarazos bioquímicos, complicaciones de los tratamientos de reproducción asistida y fallos de la implantación. También se ha postulado que las distintas estrategias de tratamiento de los mismos podrían mejorar los resultados de las técnicas de reproducción asistida, como son los tratamientos con ácido acetil salicílico (AAS), heparina de bajo peso molecular (HBPM), corticoides, hidroxicloroquina u otros.

En base a estas hipótesis, nos planteamos una serie de interrogantes en relación a la vinculación entre TH, SAF y esterilidad, y tratamientos de reproducción asistida, para lo cual realizamos una búsqueda bibliográfica para conocer la mejor evidencia disponible actualmente, así como las recomendaciones a nivel internacional para establecer en qué casos se debería indicar el estudio de TH y SAF, y en qué casos se debería indicar tratamiento.

Ante la ausencia de causa aparente o en pacientes con fracaso en las técnicas de reproducción asistida, se ha postulado que los trastornos de la coagulación (trombofilia hereditaria y adquirida) podrían ser una causa de los mismos, pero de la revisión de la literatura se concluye que no hay una clara relación causal demostrada, y a su vez dada la heterogeneidad de los resultados no es posible concluir que el tratamiento profiláctico en la TH mejore el pronóstico reproductivo de mujeres sometidas a FIV.

\section{Trombofilia hereditaria}

Los estudios que intentan relacionar infertilidad o fallos en los tratamientos de reproducción asistida con TH tienen como limitante que son estudios observacionales y que muchos de ellos no ajustan las variables potencialmente confundentes, todo lo cual determina que sus resultados no puedan considerarse en la mayoría de los casos concluyentes. Es importante en tal sentido analizar el conjunto de la evidencia disponible y la tendencia general y dirección de los resultados, antes que los hallazgos puntuales de una investigación en particular.

Se ha planteado que la presencia de TH podría generar más embarazos bioquímicos o abortos espontáneos precoces, inclusive luego de tratamientos de fertilización in vitro (FIV).

Frente a la pregunta de si hay asociación entre TH y esterilidad, o eficacia de la FIV, se plantean los siguientes estudios. En 2003, Martinelli et al. publicaron un estudio de caso control, comparando la frecuencia de TH en 234 mujeres sometidas a FIV (162 mujeres que no lograron embarazo y 72 que se embarazaron y 234 mujeres que concibieron espontáneamente) y no encontraron diferencia estadísticamente significativa en los grupos ${ }^{1}$. En 2006, Coulam et al. reportaron la prevalencia de TH de 10 genes en 42 mujeres con historia de fracaso de transferencia embrionaria posfecundación in vitro, con la de 20 mujeres fértiles; más de tres 
mutaciones fueron encontradas en el $74 \%$ de las mujeres con falla implantatoria y en el $20 \%$ de los controles ${ }^{2}$. En el mismo año Qublan et al. reportaron la frecuencia de TH en 90 mujeres con al menos tres fallas de FIV, con la de 90 mujeres que consiguieron embarazo luego del primer intento de FIV y 100 mujeres que concibieron espontáneamente y sin antecedentes de aborto; la frecuencia de trombofilia en el primer grupo fue de $69 \%$, en el segundo de $26 \%$ y en tercero de $25 \%{ }^{3}$. Nisio et al. en 2018 analizan la influencia de las TH y concluyen que la probabilidad de nacido vivo no se modifica por la presencia de TH. No hubo asociación individual ni múltiple de las TH con la tasa de embarazo clínico ni test de embarazo positivo. No hubo trombosis venosas ni tromboembolias durante el estudio. Por todo esto concluyen que no hay asociación estadística que implique mayor riesgo durante la FIV ${ }^{4}$. Xiaofang Tan et al. en 2016 realizaron un meta-análisis buscando asociación entre resultados de FIV y las TH y no encontraron asociación en los resultados de las mismas ${ }^{5}$.

En conclusión, los estudios evaluados no demuestran que la TH sea causa de esterilidad o se asocie a fallos reiterados de FIV ya que la mayoría son determinaciones de prevalencia y no es posible determinar la temporalidad de la asociación trombofilia e infertilidad. Por otra parte, la población en la que se determinó la frecuencia de trombofilia es diferente entre los estudios. En general la prevalencia de trombofilia varió entre un $43 \%$ y un $74 \%$.

Frente a la pregunta de si el tratamiento con HBPM y/o AAS tuvieran eficacia en aquellas pacientes con esterilidad y FIV y portadoras de TH, se plantean los siguientes trabajos. Se han publicado estudios que intentan probar la eficacia de tratar a las pacientes con TH que van a someterse a FIV. Se evalúo la utilización de AAS y HBPM con resultados contradictorios. Stern et $\mathrm{al}^{6}$ incluyeron pacientes con el antecedente de haberse transferido al menos 10 embriones sin lograr embarazo y administraron AAS asociada a HBPM y encontraron en el grupo tratado un $15 \%$ de embarazo, similar al $18 \%$ del grupo placebo 6 . Por otro lado, que Qublan et al. Incluyeron mujeres con al menos tres ciclos de FIV sin lograr embarazo en los que se le administró HBPM y hallaron un $31 \%$ vs $10 \%$ en el grupo placebo de embarazos exitosos ${ }^{3}$. Ninguno de estos estudios consideró otros factores pronósticos como la edad de la mujer, el número de embriones transferidos y el estadio de desarrollo embrionario. La tasa de embarazo por transferencia embrionaria en los grupos tratados también fue distinta en ambos estudios. Considerando esto, sumado a la heterogeneidad de los resultados, no es posible concluir que el tratamiento profiláctico en la TH mejore el pronóstico reproductivo de mujeres sometidas a FIV.

La Sociedad Americana de Medicina Reproductiva (ASRM) luego de analizar más de 2000 pacientes emitió sus recomendaciones, sugiriendo que no hay indicación de buscar de rutina trombofilias hereditarias previo a una FIV, y que el tratamiento de las mismas no cambia el resultado de la misma?.

\section{Síndrome antifosfolípidico}

A pesar de que la infertilidad no es parte de los criterios clínicos de SAF, en la práctica diaria en nuestro país y la región es frecuente que se soliciten el estudio de los mismos, en pacientes que presentan esterilidad o que van a someterse a tratamientos de reproducción asistida.

En este capítulo se analizará la evidencia disponible de la presencia de anticuerpos antifosfolipídicos (AAF) o SAF en relación a infertilidad y técnicas de reproducción asistida.

Si bien se ha planteado que los AAF podrían afectar la invasión trofoblástica y decidualización uterina, y se ha hipotetizado que dichos anticuerpos podrían afectar incluso antes que se diagnostique el embarazo, esto nunca ha podido ser confirmado ${ }^{8,9}$.

En los últimos años se han realizado múltiples estudios intentando vincular los AAF con la infertilidad, y para ello se ha investigado dicha asociación en distintos subgrupos de pacientes: infertilidad de causa inexplicada, pacientes candidatas a FIV, pacientes con fracaso de la FIV. La mayoría de los autores destacan que existe mucha disparidad entre los estudios publicados, desde las definiciones de la infertilidad, la esterilidad de causa inexplicada y los criterios por los que llevar a dicho diagnóstico y los criterios utilizados para definir SAF ${ }^{8}$.

Levy et al. en 2015 publicaron una revisión sistemática de la literatura buscando estudios que evalúan la presencia de AAF en mujeres infértiles y estudiaron la asociación entre éstos y el resultado de la FIV, así como los efectos del tratamiento de los mismos en estas pacientes ${ }^{9}$. Se incluyeron 29 estudios, en los cuales la frecuencia de los AAF era mayor que lo reportado en la población general. Sin embargo, observaron que en muchos estudios, las pacientes no cumplían con los criterios para el screening de los mismos, ni el nivel de corte utilizado era el recomendado (considerado significativo). La presencia de dichos anticuerpos no tuvo influencia en el resultado de la FIV en la mayoría de los estudios analizados, similar a los datos ya publicados en meta-análisis previos ${ }^{9}$. Los autores remarcan que el tratamiento de estas pacientes durante 
la FIV no mejora la tasa de embarazo y por tanto concluyen que no existe evidencia que apoye la búsqueda de AAF en pacientes con infertilidad, o el tratamiento de las mismas con el objetivo de mejorar los resultados de la FIV ${ }^{9}$.

En otra revisión sistemática en 2017 publicada por Chighizola et al. estudiaron la relación entre AAF y fertilidad donde concluyen que si bien hay trabajos en los cuales la prevalencia de AAF es mayor en mujeres infértiles, los trabajos que lo muestran, tienen diferentes definiciones de infertilidad, así como diferentes puntos de corte para considerar AAF positivos ${ }^{10}$.

Nisio et al. en 2018 analizan la influencia SAF en los resultados de FIV en una población de 684 mujeres y demuestran que la presencia de AAF se asocia a una mayor tasa de abortos, pero la probabilidad de nacido vivo no se modifica por la presencia de $\mathrm{AAF}^{4}$. La presencia de anticoagulante lúpico se asoció a mayor riesgo de aborto. No hubo diferencias estadísticamente significativas en cuanto a la tasa de embarazo clínico o de test de embarazos positivos ${ }^{4}$.

Recientemente, Hasbani et al. publicaron en Lupus una revisión de SAF en que plantean que 8 a $20 \%$ de las esterilidades sin causa aparente podrían tener vinculación con la presencia de AAF, aunque refieren que no hay evidencia suficiente que justifique la búsqueda sistemática de los mismos en todas las parejas que consultan por esterilidad ${ }^{11}$.

Simopoulou sugiere que la presencia de AAF no tiene influencia en los resultados de embarazo luego de los tratamientos de reproducción asistida. Refiere que con la calidad de la evidencia actual no se podría realizar un meta-análisis porque las conclusiones generarían más confusión. Reafirman que estas pacientes presentan mayores tasas de aborto y confirman la seguridad de realizar la FIV en pacientes con $\mathrm{AAF}^{12}$.

Dichas revisiones sistemáticas y estudios están en línea con las recomendaciones de la ASRM que sugiere que no hay evidencia para solicitar AAF en pacientes con esterilidad o que se van a realizar terapias de reproducción asistida. Dichas recomendaciones se mantienen desde el 20087.

Con respecto al éxito terapéutico de pacientes con AAF y/o SAF que presentan fallos recurrentes de la implantación existen numerosas publicaciones. Algunos autores han sugerido que el tratamiento con HBPM y/o corticoides podría mejorar las tasas de implantación.

Franasiak y Scott en 2017 realizan una revisión de la evidencia disponible hasta el momento y concluyen que el uso de AAS y HBPM en los fallos de implantación no ha demostrado mejorar los resultados y que su administración en pacientes con trombofilia y RIF es empírica ${ }^{13}$.

Siristatidis et al. evaluaron en un estudio de cohorte, la administración de prednisolona y HBPM en pacientes con fallo de implantación en 115 pacientes con RIF y observaron que las tasas de embarazo clínico fueron similares, así como las tasas de aborto, y de nacidos vivos, por lo cual concluyen que la administración de prednisolona y HBPM en pacientes con RIF no mejorar los resultados en tasas de embarazo ${ }^{14}$. El mismo grupo (Siristatidis et al.) en otro trabajo compararon el efecto de la administración de HBPM en pacientes con fallo de dos o más ciclos de FIV, en 6 centros, donde estudiaron 230 pacientes y compararon las características de los ciclos y los resultados, tasas de embarazo clínico y de aborto. No encontraron diferencias estadísticamente significativas entre los dos grupos ni en tasa de embarazo clínico ni de aborto. Por lo tanto, no encontraron evidencia que apoye la administración reglada de HBPM en pacientes con fallo de dos o más ciclos de FIV ${ }^{15}$.

Las guías de la Sociedad Británica de Fertilidad realizan una revisión sobre la utilización de inmunoglobulinas en pacientes que van a someterse a una FIV. No encuentran evidencia que justifique el uso ni la seguridad de las inmunoglobulinas en pacientes con fallo de implantación que vayan a hacer una nueva FIV. Destacan los potenciales efectos adversos de las mismas como son: cefalea, náuseas, fatiga, anafilaxis e infección, entre otras complicaciones ${ }^{16}$. Adicionalmente analizan la evidencia disponible para el uso de AAS y HBPM y concluyen que tampoco hay evidencia suficiente para el uso de AAS, ni de $\mathrm{HBPM}^{16}$.

En el mismo sentido, se realizó una revisión Cochrane donde se destaca que los estudios que encontraron sugieren que la HBPM usada peri-implantación en ciclos de reproducción asistida mejoraban las tasas de nacidos vivos, eran estudios con bajo número de pacientes, heterogéneos y sensibles al modelo estadístico elegido. Señalan algunos efectos secundarios poco importantes como equimosis y sangrados. Concluyen que no se justifica el uso de HBPM con esta indicación, salvo en el marco de estudios de investigación, bien concebidos metodológicamente ${ }^{17}$. Esto es retomado por la ASRM donde ratifican que salvo estudios de investigación no se justificaría el uso de HBPM con el objetivo de mejorar los resultados de FIV, 
y que se requerirían buenos estudios para demostrar un eventual efecto de la HBPM a nivel local uterino durante los tratamientos de reproducción asistida ${ }^{17}$.

Un meta-análisis publicado por Yang et al. que incluyó 5 estudios con 935 pacientes y donde analizaron la eficacia de la HBPM en los resultados de FIV ICSI en pacientes sin trombofilias no se hallaron diferencias estadísticamente significativas en cuanto a tasa de nacidos vivos, tasas de embarazo clínico y de aborto ${ }^{18}$.

La ASRM recomienda no realizar la búsqueda rutinaria de AAF en mujeres con esterilidad ni previo a una FIV y sugiere que el tratamiento de las mismas no cambia el resultado de la misma.

\section{Recomendaciones}

No se recomienda solicitar TH en forma sistemática en mujeres con esterilidad o que van a someterse a técnicas de reproducción asistida.

No se recomienda realizar tratamiento con AAS o HBPM con el objetivo de mejorar los resultados obstétricos en mujeres con TH con esterilidad o que van a someterse a técnicas de reproducción asistida.

No se recomienda solicitar AAF en forma sistemática en mujeres con esterilidad o que van a someterse a técnicas de reproducción asistida.

No se recomienda realizar tratamiento con AAS o HBPM con el objetivo de mejorar los resultados obstétricos en mujeres con AAF con esterilidad o que van a someterse a técnicas de reproducción asistida.

No se recomienda el uso de gamaglobulinas u otros fármacos (hidroxicloroquina, prednisona) en pacientes con esterilidad o previo a FIV.

\section{Bibliografía}

1- Martinelli I, Taioli E, Ragni G, Levi-Setti P, Passamonti SM, Battaglioli T, et al. Embryo implantation after assisted reproductive procedures and maternal thrombophilia. Haematologica. 2003;88(7):789-793.

2- Coulam CB, Jeyendran RS, Fishel LA, Roussev R. Multiple thrombophilic gene mutations are risk factors for implantation failure. Reprod. Biomed. Online. 2006;12(3):322-327.

3- Qublan HS, Eid SS, Ababneh HA, Amarin ZO, Smadi AZ, AI-Khafaji FF, et al. Acquired and inherited thrombophilia: implication in recurrent IVF and embryo transfer failure. Hum. Reprod. 2006;21(10):2694-8.

4- Di Nisio M, Ponzano A, Tiboni GM, Guglielmi MD, Rutjes AWS, Porreca E. Effects of multiple inherited and acquired thrombophilia on outcomes of in-vitro fertilization. Thromb Res. 2018 Jul;167:26-31. doi: 10.1016/j.thromres.2018.05.006.

5- Tan X, Yu Z, Sao J, Chen L, Shen Y, Ding J, et al. Association between in vitro fertilization outcomes and inherited thrombophilias: a meta-analysis. J. Assist. Reprod. Genet. 2016;33(8):1093-1098.

6- Stern C, Chamley L, Norris H, Hale L, Baker HWG. A randomized, double-blind, placebo-controlled trial of heparin and aspirin for women with in vitro fertilization implantation failure and antiphospholipid or antinuclear antibodies. Fertil. Steril. 2003;80(2):376-383.

7- Practice Committee of American Society for Reproductive Medicine. Anti-phospholipid antibodies do not affect IVF success. Fertil Steril. 2008 Nov;90(5 Suppl):S172-3. doi: 10.1016/j.fertnstert.2008.08.100.

8- Chighizola CB, De Jesus GR. Antiphospholipid antibodies and infertility. Lupus. 2014;23(12):12321238.

9- Levy RA, Dos Santos FC, de Jesús GR, de Jesús NR. Antiphospholipid Antibodies and Antiphospholipid Syndrome during Pregnancy: Diagnostic Concepts. Front Immunol. 2015 May 7;6:205. doi: 10.3389/fimmu.2015.00205

10- Chighizola CB, Raimondo MG, Meroni PL. Does APS Impact Women's Fertility? Curr Rheumatol Rep. 2017 Jun;19(6):33. doi: 10.1007/s11926-017-0663-7.

11- El Hasbani G, Khamashta M, Uthman I. Antiphospholipid syndrome and infertility. Lupus. 2020;29(2):105-117.

12- Simopoulou M, Sfakianoudis K, Maziotis E, Grigoriadis S, Giannelou P, Rapani A, et al. The impact of autoantibodies on ivf treatment and outcome: A systematic review. Int. J. Mol. Sci. 2019;20(4). 
13- Franasiak JM, Scott RT. Contribution of immunology to implantation failure of euploid embryos. Fertil. Steril. 2017;107(6):1279-1283.

14- Siristatidis C, Dafopoulos K, El-Khayat W, Salamalekis G, Anifandis G, Vrantza T, et al. Administration of prednisolone and low molecular weight heparin in patients with repeated implantation failures: a cohort study. Gynecol. Endocrinol. 2018;34(2):136-139.

15- Siristatidis C, Dafopoulos K, Salamalekis G, Galazios G, Christoforidis N, Moustakarias T, et al. Administration of low-molecular-weight heparin in patients with two or more unsuccessful IVF/ICSI cycles: a multicenter cohort study. Gynecol. Endocrinol. 2018;34(9):747-751.

16- Nardo LG, El-Toukhy T, Stewart J, Balen AH, Potdar N. British Fertility Society Policy and Practice Committee: Adjuvants in IVF: Evidence for good clinical practice. Hum. Fertil. 2015;18(1):2-15.

17- Akhtar MA, Sur S, Raine-Fenning N, Jayaprakasan K, Thornton JG, Quenby S. Heparin for assisted reproduction. Cochrane Database Syst Rev. 2013 Aug 17;(8):CD009452. doi: 10.1002/14651858. CD009452.pub2.

18- Yang XL, Chen F, Yang XY, Du GH, Xu Y. Efficacy of low-molecular-weight heparin on the outcomes of in vitro fertilization/intracytoplasmic sperm injection pregnancy in non-thrombophilic women: a metaanalysis. Acta Obstet. Gynecol. Scand. 2018; 97(9):1061-1072.

\section{Aporte de cadla autor al trabajo}

Virginia Chaquiriand: Concepción y diseño del trabajo, recolección de datos, redacción y revisión crítica del manuscrito. 016. AA 